\title{
Diffuse-type giant cell tumor of the tendon sheath in the temporal region incidentally diagnosed due to a temporal tumor: A report of two cases and review of the literature
}

\author{
JIA RUO QIN ${ }^{1,2}$, LONG JIN $^{1}$, KONG LIANG LI ${ }^{1,2}$, SHAN SHAN ZHANG ${ }^{1,2}$, JIE KONG $^{1}$ and HONG YU YANG ${ }^{1}$ \\ ${ }^{1}$ Department of Oral and Maxillofacial Surgery, Shenzhen Hospital, Peking University, Shenzhen, Guangdong 518036; \\ ${ }^{2}$ Graduate Department, Shantou University Medical College, Shantou, Guangdong 515041, P.R. China
}

Received August 6, 2014; Accepted April 29, 2015

DOI: $10.3892 / 01.2015 .3288$

\begin{abstract}
Diffuse-type tenosynovial giant cell tumor (D-GCTS) is a rare benign lesion that not only frequently occurs in the fingers, but also along the tendon sheaths of the foot and ankle. The present study reports the cases of two middle-aged patients that were diagnosed with D-GCTS. The presentation of the D-GCTS lesions was extremely rare, as the tumors were located in the temporal fossa and threatened the skull base and external auditory canal. There were similarities and differences between the two patients in their clinical symptoms, disease progressions and invading sites. The patients' disease course occurred unnoticed with the absence of pain, was protracted and became infiltrative. However, the female patient was admitted to the hospital due to the occurrence of pain in the left temporal region, and the male patient presented at the doctor due to a painless left temporal mass and external auditory canal bleeding. Therefore, the operation area of the two patients was not the same. This type of illness should be considered in the differential diagnosis for masses occurring in the temporal region. Total tumor removal is the best treatment for D-GCTS, and the careful monitoring of recurrence can achieve a good clinical outcome subsequent to the surgical resection.
\end{abstract}

\section{Introduction}

Giant cell tumor of the tendon sheath (GCTS) was initially described by Chassaignac in 1852 (1), and the lesion was defined as a malignant tumor at that time (2). Subsequently, Heurteux produced a detailed description of GCTS (3). It was found that the disease occurs more frequently in

Correspondence to: Professor Hong Yu Yang, Department of Oral and Maxillofacial Surgery, Shenzhen Hospital, Peking University, 1120 Lian Hua Road, Shenzhen, Guangdong 518036, P.R. China E-mail: hyyang192@hotmail.com

Key words: diffuse type tenosynovial giant cell tumor, the temporal fossa, skull base, external auditory canal, clinical and pathological middle-aged women compared with men (4). According to the position (large or facet joint; intra- or extra-articular), characteristics (benign or malignant) and growth pattern (diffuse or localized) of the tumor, GCTS may be classified as one of four types: Localized-type tenosynovial giant cell tumor (L-GCTS); diffuse-type tenosynovial giant cell tumor (D-GCTS); pigmented villonodular synovitis (PVNS) $(5,6)$; and malignant tenosynovial giant cell tumor (M-GCTS) $(7,8)$.

D-GCTS demonstrates invasive growth in the local region, which is considerably different from L-GCTS. D-GCTS usually develops in the synovium, and often invades the surrounding knee and soft tissue $(2,9)$. Since it arises mainly from the soft tissue outside the joints, Weiss et al (10) regarded D-GCTS as a type of PVNS that appears in the soft tissue outside the joints, and also presents with intra-articular lesions $(2,5)$. L-GCTS mainly occurs in the small joints, including the hand, foot and ankle. Image findings in previous studies reveal these soft tissue masses occur beside small joints $(9,11)$. If the results of image analysis demonstrate that the lesions have invaded the joints, and if there is a concurrent weak signal of T1W and T2W on a magnetic resonance imaging (MRI) scan, D-GCTS should be considered as a probable diagnosis (12-15).

GCTS usually occurs in individuals between the ages of 30 and 50 years (5). As a painless and slow-growing disease, it has been termed an innocent tumor $(4,5,13)$. However, it may recur if incompletely removed (5). D-GCTS has a low incidence of two per 1,000,000 per year in middle-young aged person (5). With a pain-free and long clinical history, surgery is the standard treatment. With the risk of recurrence, rare cases may become M-GCTS $(5,7,13)$.

D-GCTS mainly occurs in weight-bearing joints, such as the knee, ankle and foot, and a small number of lesions occur in non-weight-bearing joints (16). D-GCTS of the tendon sheath in the temporal region is extremely rare $(8,17,18)$.

The present study reports two cases, consisting of the case of a 33-year-old female patient that presented with D-GCTS of the temporal fossa, which had invaded the skull base, and the case of a 30-year-old male patient that 

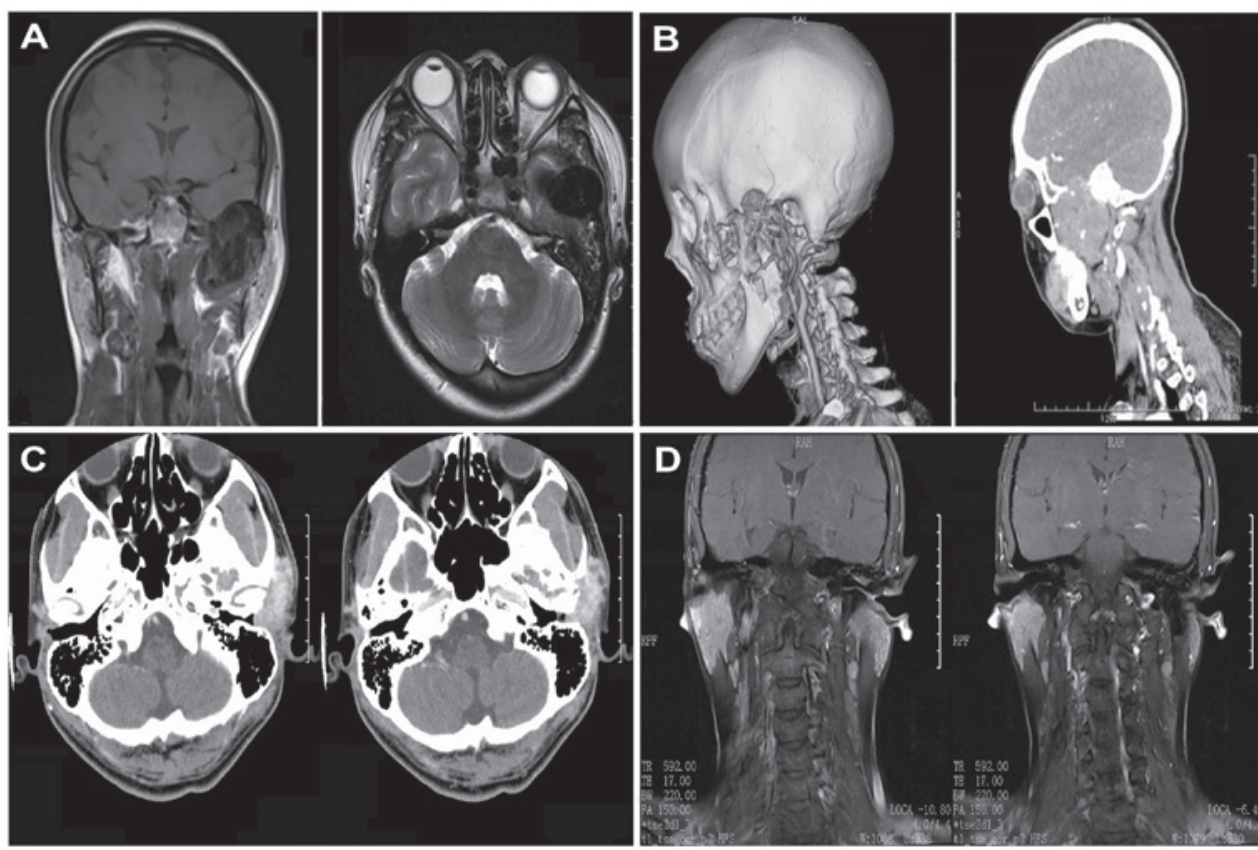

Figure 1. (A) CT performed on the female patient, revealing the destruction of the nidus in left sphenoid wing and skull base bones. (B) MRI of the female patient, revealing the nidus. (C) CT examination of the male patient to identify the lesion using a plain scan. (D) MRI examination of the male patient, revealing the lesion on a T1-weighted image of the coronal plane, using an enhanced scan. CT, computed tomography; MRI, magnetic resonance imaging.

presented with D-GCTS that had invaded the external auditory canal.

\section{Case report}

A 33-year-old woman who first felt pain in September 2009 in the left temporomandibular joint pain and limitation of mouth opening subsequent to biting a hard substance three years prior to presentation underwent a lateral skull X-ray examination at the Department of Oral of Bao'an District Hospital of traditional Chinese medicine (Shenzhen, Guangdong, China), and was diagnosed with temporomandibular joint dysfunction syndrome (TMDS). The patient possessed no systemic disease and no associated family medical history.

For almost one year prior to October 10, 2012, the patient had experienced pain in the temporomandibular region when tired or subsequent to chewing for an extended period, but had not received treatment. The patient experienced an increase in the pain in the left temporal region, without limited movement of the joint in July 2012. Subsequent to the imaging examination performed in the Department of Oral of Bao'an District Hospital of traditional Chinese medicine, the destruction of the left temporal bone was revealed, in addition to the presence of soft tissue space-occupying lesions in the deep left buccal division. The patient was then referred to the Department of Oral and Maxillofacial Surgery of Shenzhen Hospital, Peking University (Shenzhen, Guangdong, China).

The clinical examination revealed that the left temporal bone was slightly more ridged compared with the right temporal bone. The oral mucosa, tongue activity, opening of mouth and lymph nodes in associated regions were all determined to be normal.

Subsequently, a 30-year-old man was referred to the Department of Oral and Maxillofacial Surgery of Shenzhen
Hospital, Peking University, with a painless left temporal mass and external auditory canal bleeding. The patient found the mass incidentally in April 2013. Since the lesion was painless and had no influence on daily life, the patient overlooked the mass prior to the commencement of bleeding of the external auditory canal. The patient underwent a biopsy due to a rupture in the external auditory canal. The biopsy results suggested that the lesion may be a left plane epidermoid cyst associated with infection.

Similarly to the female patient, the male patient possessed no systemic disease and no relevant family history. The clinical examination expressed the same findings as the examination performed on the female patient, with the exception of the bleeding in the external auditory canal.

The two patients were admitted to hospital and were diagnosed with a left temporal tumor.

The results of computed tomography (CT) and spiral CT performed on the female patient revealed the mass lesion in the left temporal fossa and the destruction of the left temporal, sphenoid wing and skull base bones. Magnetic resonance imaging (MRI) revealed that the mass lesion under the left temporal fossa and skull base in this patient broke through the left temporal bone to push the left temporal lobe. The lesion also pushed the medical and lateral pterygoid muscle. The range of destruction and absorption of the left temporal and sphenoid wing bone was $\sim 6.2 \times 3.8 \mathrm{~cm}$. The lesion exhibited characteristic hypointense and heterogeneous hypointense signals on T1W and T2W images (Fig. 1).

Subsequent to the CT and MRI examination performed on the male patient, the results revealed that the lesion occurred in the left temporal fossa and extended into the external auditory canal. The junction of the zygomatic arch and temporal bone exhibited signs of destruction, and the report indicated that a diagnosis of malignant tumor should be considered (Fig. 1). 
A

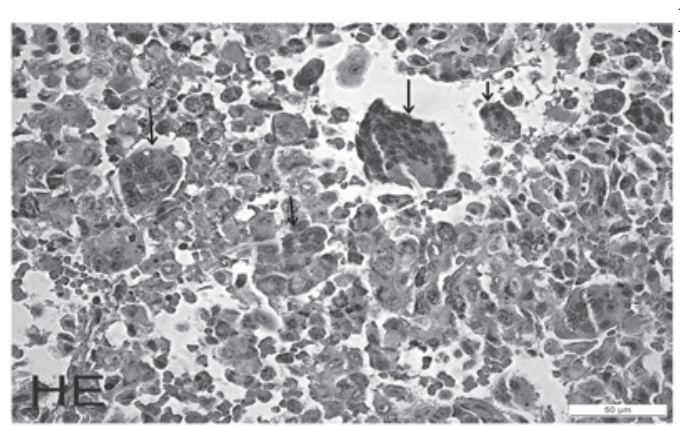

Bb

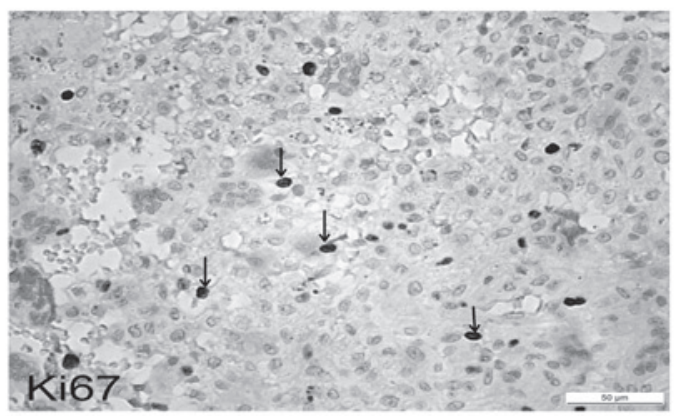

Bd

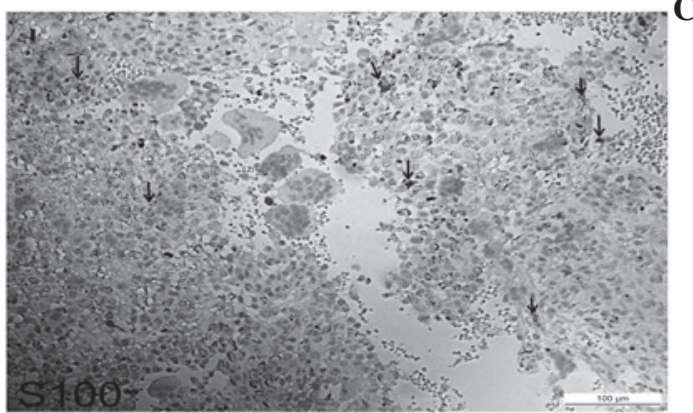

D

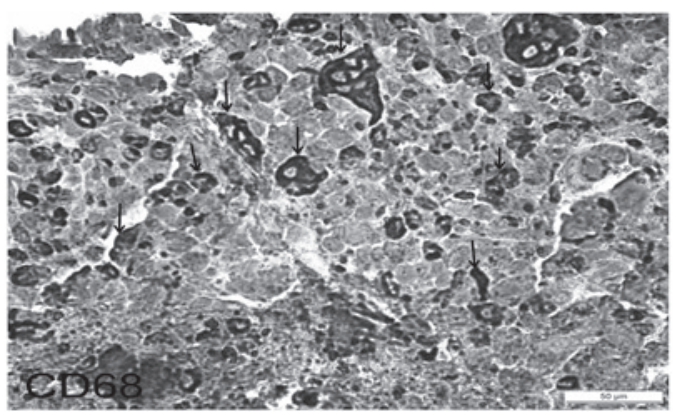

Ba
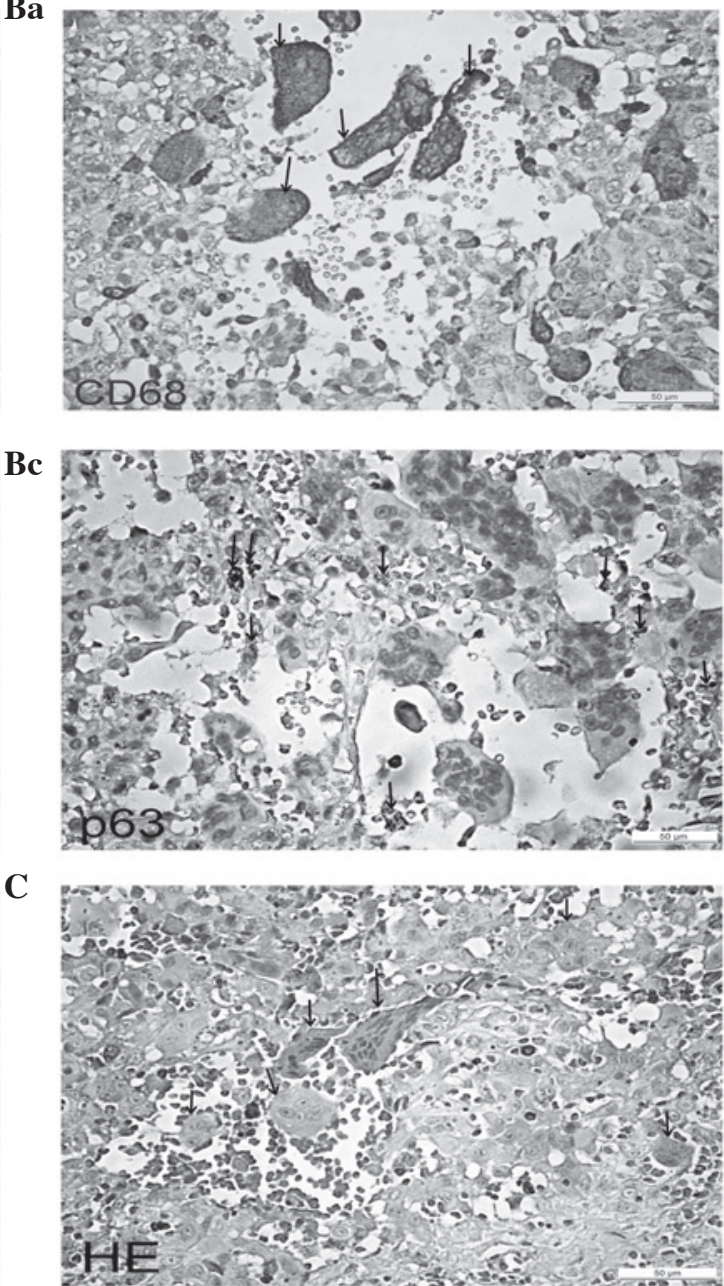

Figure 2. (A) Result of post-operative pathological examination of the tumor tissue obtained from the female patient, and (B) immunohistochemical staining of the tissue section. (C) Result of post-operative pathological examination performed on the tumor tissue obtained from the male patient, and (D) immunohistochemical staining of the tissue section.

The female patient underwent expanded lesion sites resection of the neoplasm in the left temporal fossa and skull base, osteotomy of the left mandible and resection of the left zygomatic arch. Internal fixation with a titanium plate was performed, along with transmyocardial revascularization of the zygomatic arch. Subsequently, the temporalis myofascial flap and adjacent gingival flap was used to repair the surgical defect. The lesion excised during the procedure was $\sim 1.6 \times 1.6 \mathrm{~cm}$, and the edges of the resection margin did not contain tumor cells.

The male patient underwent expanded lesion sites resection of the left temporal neoplasm and decompression of the facial nerve. Subsequently, anastomosis of the facial nerve was conducted to rebuild the function of facial nerve. The external auditory canal was then repaired and the surgical defect was repaired with the opisthotic free flap and the temporalis myofascial flap. The neoplasm that developed in the external auditory canal had been removed at the same time. The lesion removed during the procedure was $\sim 2.5 \times 2.0 \times 1.5 \mathrm{~cm}$ in size, and the edges of the resection margin were negative for tumor cells.

Post-operative pathological examination of the resected specimen from the female patient revealed that the tissue section was rich in giant cells. The cells were round or polygonal in shape, and numerous cells with a clear perimeter were not mature. Chondroblasts accompanied by individual or several multi-core osteoclastic-like giant cells were identified under 
microscopy (x400 magnification, Leica DM4000B). In addition, the immunohistochemical staining for the section of the lesion revealed that the cells did not express cytokeratin, and were weakly posistive for cluster of differentiation (CD)68, S-100 and cytoplasmic p63. In total, 5-10\% of cells stained for Ki-67 expression. Microscopically, the cell population mostly consisted of single nuclear cells and multinucleated giant cells, and the prescence of multifocal irregular calcification regions within pane-like calcification was common. The shape of cells remained well-differentiated, and mitotic structures were rarely identified (Fig. 2).

The histopathological examination on the tumor excised from the male patient provided reasonable support for the diagnosis of D-GCTS, which was the same diagnosis made for the female patient. The results for the male patient of histopathological examination and immunohistochemical staining are listed as follows. The section of mass of the left temporal region revealed that histocytes and multinucleated giant cells constituted the tumor tissue. Cartilage tissue was also present. The immunohistochemical staining identified that the three immunological indicators of the section were CD68(+), S-100(-) and CD1a(-) (Fig. 2).

\section{Discussion}

The clinical manifestations of D-GCTS are local swelling, limitation of movement and joint closure (19). The incidence of D-GCTS is observed more often in female patients compared with male patients $(4,5)$. The age at onset of patients with D-GCTS is, overall, younger compared with the age at onset of patients with L-GCTS, but the course of the disease is much longer. Compared with the common pathogenic sites of L-GCTS, weight-bearing joints may be involved in D-GCTS. However, it is extremely infrequent that the lesion involves the temporal fossa $(8,18)$. For this reason, the correct diagnosis of the disease is a considerable challenge $(2,9,13)$. Additional auxiliary examinations should be performed, and detailed information from patients should be recorded, which is vital for the confirmation of a therapeutic schedule $(4,5,13,15)$.

In imaging examinations, X-rays may reveal joint capsular swelling or the increased density of circular shadow, and also reveal the defect, hardening and hyperplasia at the edge of the joint, where the lesions erode the articular surface $(13,20,21)$. When the lesions extend intra-articularly, the X-ray may reveal a widened joint space and oppressive involvement in the adjacent bone cortex (13). CT demonstrates an advantage in revealing the extent of disease, particularly in the bone cortex. The differences between D-GCTS and osteosarcoma or chondrosarcoma may be determined from the CT results $(17,21)$, as diffuse thickening of soft tissues around the joints, oppressive absorption of the affected bones, the presence of a clear boundary, the lack of periosteal reaction and the presence of a soft tissue mass also without calcification or ossification may be observed on CT $(13,21,22)$. MRI has an extremely high sensitivity and specificity for the diagnosis of G-GCTS $(16,23)$. MRI can reveal the diffuse and invasive growth of the irregular mass lesion adjacent to the joints, the origin and scope of the lesions, and the level of intra-articular invasion. Generally the MRI signal is weak and this is one of the characteristics of the imaging diagnosis of D-GCTS $(12,19,21)$.

The cell components of D-GCTS include the large synovial-like mononuclear, small histiocytoid, foamy histiocyte and inflammatory cells, fibroblasts and the osteoclast-like multinucleated giant cells $(5,24,25)$. The number of large synovial-like mononuclear cells that are positive for clusterin in D-GCTS are more than that in L-GCTS $(\mathrm{P}<0.01)$ and PVNS $(\mathrm{P}<0.05)(26)$.

D-GCTS is challenging to diagnose correctly as the lesions may invade the temporal fossa and extend to the external auditory canal or skull base (18). D-GCTS should be distinguished from temporomandibular joint dysfunction syndrome, synovial sarcoma, xanthomatous inflammatory malignant fibrous histiocytoma and M-GCTS $(2,5,13)$. Occasionally, the clinical symptoms and imaging examination does not result in a final precise diagnosis; therefore, the post-operative histopathological examination is the gold standard for the confirmation of diagnosis (9).

The pathogenesis of D-GCTS has not been clarified, and certain studies have concluded that the cause of disease is associated with bleeding subsequent to trauma and lipid metabolism disorders $(5,13,15)$.

D-GCTS in the temporal fossa may result in the limitation of the activity of the temporomudibular joint, difficulty in opening the mouth, local pain and numerous notable consequences when the lesion extends into the cranial cavity. Therefore, early detection and treatment possesses a considerable significance. In addition, due to the high recurrence rate, complete surgical treatment and post-operative follow-up review is required $(11,14)$.

\section{References}

1. Chassaignac M: Cancer de la gaîne des tendons. Gazette Hôpital Civil Militaire 47: 2, 1852 (In French).

2. Stevenson JD, Jaiswal A, Gregory JJ, Mangham DC, Cribb G and Cool P: Diffuse pigmented villonodular synovitis (diffuse-type giant cell tumour) of the foot and ankle. Bone Joint J 95-B: 384-390, 2013.

3. Heurtaux MA: Myelome des gaines tendineuses Arch Gen Med 167: 40-54, 1891 (In French).

4. Di Grazia S, Succi G, Fraggetta F and Perrotta RE: Giant cell tumor of tendon sheath: Study of 64 cases and review of literature. G Chir 34: 149-152, 2013.

5. van der Heijden L, Gibbons CL, Dijkstra PD, et al: The management of diffuse-type giant cell tumour (pigmented villonodular synovitis) and giant cell tumour of tendon sheat (nodular tenosynovitis). J Bone Joint Surg Br 94: 882-888, 2012.

6. Caputo V, Fiorella S and Orlando E: Postsurgical paracicatricial cutaneous satellitosis of giant cell tumour of the tendon sheath, localized type. Case Rep Dermatol 3: 118-123, 2011.

7. Theunissen CI, Bras J, Lienden KP and Obdeijn MC: Malignant giant cell tumor in the carpal tunnel: a case report and review of literature. J Wrist Surg 2: 271-275, 2013.

8. Leonard J, Gökden M, Kyriakos M, Derdeyn CP and Rich KM: Malignant giant-cell tumor of the parietal bone: Case report and review of the literature. Neurosurgery 48: 429-429, 2001.

9. Somerhausen NS and Fletcher CD: Diffuse-type giant cell tumor clinicopathologic and immunohistochemical analysis of 50 cases with extraarticular disease. Am J Surg Pathol 24: 479-492, 2000.

10. Weiss SW, Goldblum JR and Enzinger FM: Benign tumors and tumor-like lesions of synovial tissue. In: Enzinger and Weiss's Soft Tissue Tumors. 5th edition. Elsevier, Philadelphia, PA, USA, pp20, 2007.

11. Kotwal PP, Gupta V and Malhotra R: Giant-cell tumour of the tendon sheath: Is radio therapy indicated to prevent recurrence after surgery? J Bone Joint Surg 82: 571-573, 2000. 
12. Kim KW, Han MH, Park SW, et al: Pigmented villonodular synovitis of the temporomandibular joint: conventional findings in four cases. Eur J Radiol 49: 229-234, 2004.

13. Wang K, Liu X, Liu Z and Yu M: Diagnosis and treatment of primary diffuse-type tenosynovial giant cell tumors of the cervical spine. Chin Med J (Engl) 2: 791-792, 2014.

14. Nakahara H, Matsuda S, Harimaya K, et al: Clinical results of open synovectomy for treatment of diffuse pigmented villonodular synovitis of the knee: Case series and review of literature. Knee 19: 684-687, 2012.

15. Griffin AM, Ferguson PC, Catton CN, et al: Long-term outcome of the treatment of high-risk tenosynovial giant cell tumor/pigmented villonodular synovitis with radiotherapy and surgery. Cancer 118 4901-4909, 2012.

16. Gibbons CL, Khwaja HA, Cole AS, Cooke PH and Athanasou NA Giant-cell tumour of the tendon sheath in the foot and ankle. J Bone Joint Surg Br 84: 1000-1003, 2002.

17. Isaacson B, Berryhill $\mathrm{W}$ and Arts HA: Giant-cell tumors of the temporal bone: management strategies. Skull Base 19: 291-301, 2009.

18. Day JD, Yoo A and Muckle R: Pigmented villonodular synovitis of the temporomandibular joint: a rare tumor of the temporal skull base. J Neurosurg 109: 140-143, 2008.

19. Llauger J, Palmer J, Rosón N, Cremades R and Bagué S: Pigmented villonodular synovitis and giant cell tumors of the tendon sheath: Radiologic and pathologic features. AJR Am J Roentgenol 172: 1087-1091, 1999.

20. Bemporad AJ, Chaloupka JC, Putman CM, et al: Pigmented villonodular synovitis of the temporomandibular joint: Diagnostic imaging and endovascular therapeutic embolization of a rare head and neck tumor. AJNR Am J Neuroradiol 20: 159-162, 1999.
21. Murphey MD, Nomikos GC, Fleming JD, Gannon FH, Temple HT and Kransdorf MJ: Imaging of giant cell tumor and giant cell reparative granuloma of bone: Radiologic-pathologic correlation. Radiographics 21: 1283-1309, 2001.

22. Siribumrungwong K, Tangtrakulwanich B and Nitiruangjaras A: Unusual presentation of giant cell tumor originating from a facet joint of the thoracic spine in a child: A case report and review of the literature. J Med Case Reports 7: 178,2013

23. Zhao H, Maheshwari AV, Kumar D and Malawer MM: Giant cell tumor of the pes anserine bursa (extra-articular pigmented villonodular bursitis): A case report and review of the literature. Case Rep Med 2011: 491470, 2011.

24. Athanasou NA, Quinn J, Ferguson DJ and McGee JO: Bone resorption by macrophage polykaryons of giant cell tumor of tendon sheath. Br J Cancer 63: 527-533, 1991.

25. Ho CY and Maleki Z: Giant cell tumor of tendon sheath: Cytomorphologic and radiologic findings in 41 patients. Diagn Cytopathol 40 (Suppl 2): E94-E98, 2012.

26. Darling JM, Goldring SR, Harada Y, Handel ML, Glowacki J and Gravallese EM: Multinucleated cells in pigmented villonodular synovitis and giant cell tumor of tendon sheath express features of osteoclasts. Am J Pathol 150: 1383-193, 1997. 